\title{
Drug diffusion and biological responses of arteries using a drug-eluting stent with nonuniform coating
}

This article was published in the following Dove Press journal:

Medical Devices: Evidence and Research

17 March 2016

Number of times this article has been viewed

\section{Noboru Saito \\ Yuhei Mori \\ Sayaka Uchiyama}

Terumo Corporation R\&D Center, Inokuchi, Nakai-machi, Ashigarakamigun, Kanagawa, Japan
Correspondence: Noboru Saito Terumo Corporation R\&D Center, I 500 Inokuchi, Nakai-machi, Ashigarakami-gun, Kanagawa 259-0I5I, Japan

Tel $+8 \mid 465$ 8I 425 I

Fax +8I 465 8I 4217

Email noboru_saitou@terumo.co.jp
Abstract: The purpose of this study was to determine the effect of a nonuniform coating, abluminal-gradient coating (AGC), which leaves the abluminal surface of the curves and links parts of the stent free from the drug coating, on the diffusion direction of the drug and the biological responses of the artery to drug-eluting stent (DES) by comparing the AGC-sirolimus stent and the conventional full-surface coating (CFC) sirolimus stent. The study aimed to verify whether the AGC approach was appropriate for the development of a safer DES, minimizing the risks of stent thrombosis due to delayed endothelialization by the drug and distal embolization due to cracking of the coating layer on the hinge parts of the DES on stent expansion. In the in vitro local drug diffusion study, we used rhodamine B as a model drug, and rhodamine B released from the AGC stent diffused predominantly into the abluminal side of the alginate artery model. Conversely, rhodamine B released from the CFC stent quickly spread to the luminal side of the artery model, where endothelial cell regeneration is required. In the biological responses study, the luminal surface of the iliac artery implanted with the AGC-sirolimus stent in a rabbit iliac artery for 2 weeks was completely covered with endothelial-like cells. On the other hand, the luminal surface of the iliac artery implanted with the CFC-sirolimus stent for 2 weeks only showed partial coverage with endothelial-like cells. While thrombosis was observed in two of the three CFC-sirolimus stents, it was observed in only one of the three AGC-sirolimus stents. Taken together, these findings indicate that the designed nonuniform coating (AGC) is an appropriate approach to ensure a safer DES. However, the number of studies is limited and a larger study should be conducted to reach a statistically significant conclusion.

Keywords: drug-eluting stent, abluminal coating, drug diffusion, endothelialization

\section{Introduction}

Drug-eluting stents (DESs), stents coated with a mixture of antiproliferative drugs (eg, sirolimus) and excipients (eg, polymers), were introduced into clinical practice in 2002 with the aim of reducing restenosis, which, at the time, occurred in $15 \%-25 \%$ of patients, receiving bare-metal stents (BMSs), owing to proliferation of vascular smooth muscle cells (SMCs). Subsequent clinical trials investigating different types of DES confirmed their efficacy in this regard. However, late or very late stent thrombosis was reported as early as 2004, especially in patients discontinuing dual antiplatelet therapy (DAPT). Subsequently, a new issue was raised, with patients implanted with a DES being required to continue the DAPT for at least 12 months. However, patients implanted with a BMS could discontinue the DAPT only after 1 month, thus forcing the patients to not only consider the costs of the DAPT but also the bleeding risk associated with these stents. ${ }^{1-4}$ Therefore, toward the next-generation DES, a new challenge for minimization 
of stent thrombosis has begun and is currently being tackled by many device manufactures. ${ }^{5,6}$

The mechanisms behind late stent thrombosis appear to be multifactorial, varying from factors such as inappropriate stent deployment techniques to delayed or inadequate endothelialization of the stent surface. ${ }^{7-10}$ One approach to this challenge is targeting of an antiproliferative drug to the vascular SMCs activated by stenting and subsequent inflammation, rather than to the endothelial cells, which must proliferate. For this approach, the abluminal coating with sirolimus derivatives, which leaves the luminal side of the stent free from the drug and polymers, has been introduced in some of the latest $\mathrm{DESs}^{5}$ together with bioabsorbable polymers, eg, poly(lactic acid), as excipients, as the excipients polymers are considered a potential cause of the chronic inflammation and consequent endothelium dysfunction. ${ }^{711-13}$ The abluminal coating was designed with the intention to enhance reendothelialization, ie, proliferation of endothelial cells, on the luminal surface of the stent, by preventing exposure of this surface to the antiproliferative drug. Accordingly, reduced late stent thrombosis and better endothelial function of the artery implanted with DES employing the abluminal coating with sirolimus derivatives have been reported in both preclinical and clinical settings. ${ }^{14-17}$ On the contrary, no significant differences in endothelialization between the abluminal coating and the conventional full-surface coating (CFC) of DES have been reported. ${ }^{18-20}$ However, relatively little is currently known about the effects of the abluminal coating on the drug diffusion and the biological response. In addition to the abluminal coating, we recently developed a novel "gradient coating technology" for DES, which further leaves the abluminal surface of the curves and links parts of the stent free from the drug and biodegradable polymer coating by gradually decreasing the coating layer thickness toward the hinge area, ${ }^{21}$ thus preventing the coating layer from cracking on stent expansion. ${ }^{22-25}$ This drives us to the question of whether the drug released from the newly developed Ultimaster ${ }^{\mathrm{TM}}$ sirolimus-eluting coronary stent with this gradient coating (Terumo Europe, Leuven, Belgium) diffuses uniformly to the luminal surface of the artery, and there is renewed interest in the effects of the designed nonuniform abluminal coating on the drug diffusion and biological responses of the DES.

Seidlitz et al developed an in vitro evaluation method for the local drug distribution of DES. ${ }^{26,27}$ They used alginate gel and rhodamine $\mathrm{B}$ as an artery model and a model drug, respectively, and a rhodamine B-coated stent was deployed in the alginate artery model, after which the drug distribution in the stented artery was investigated by monitoring fluorescence of rhodamine B while flowing phosphate-buffered saline (PBS) in the lumen. They reported that the initially high concentration gradients in the hydrogel were decreased during further perfusion due to redistribution within the gel and diffusive washout into the perfusion liquid, and consequently, homogeneous distributions independent of the distance from the releasing strut were detected within the innermost hydrogel layer lining the lumen only after 5 minutes. In addition to these reports, Zhu et al suggested that higher circumferential vascular diffusion of a hydrophobic drug results in more uniform drug loading in the upper layers, and this can be achieved by mathematically modeling the delivery of the drug from a DES implanted in a coronary artery. ${ }^{28}$

On the other hand, O'Brien et al reported the existence of inhomogeneous and asymmetric arterial drug distributions by visualizing the resulting spatial maps of fluorescently labeled drug. They used an epifluorescence microscope with a newly developed in vitro dynamically simulated benchtop model composed of a flow channel, drug-eluting strut, and tissue-mimicking poly(vinyl alcohol). ${ }^{29}$ Moreover, Hwang et al and Balakrishanan et al investigated the drug deposition for DES by applying a coupled computational fluid dynamics and mass transfer model ${ }^{30-32}$ and reported that the drug eluted from the abluminal stent strut surface accounted for only $11 \%$ of the total deposition when all strut surfaces eluted the drug, whereas, remarkably, the drug eluted from the abluminal surface accounted for $43 \%$ of the total deposition.

The purpose of this study was to further explore the effects of the designed nonuniform abluminal coating, such as the abluminal-gradient coating (AGC), on the diffusion direction of drug and the biological responses of DES, and to verify if this approach, which includes targeting the drug to the luminal side of the stented artery with a nonuniform coating, is appropriate to ensure safer DES. We first prepared rhodamine B-eluting stents with AGC and CFC. Subsequently, we investigated the diffusion direction of the drug in a stented artery from both the circumferential and longitudinal directions. To prove our hypothesis that drug released from AGC diffuses predominantly to the luminal side of the stented artery but uniformly spreads over the luminal side in in vitro, we used rhodamine B as a fluorescence tracer and an alginate artery model in place of sirolimus and an actual artery. We assumed that the diffusion direction of rhodamine B in the artery model reflects the diffusion directions of sirolimus in the stented artery; however, the diffusion coefficient of hydrophilic rhodamine $\mathrm{B}$ in alginate gel without binding sites is different 
from that of hydrophobic sirolimus in the actual artery with binding sites. Also, the time frame for diffusion of rhodamine $\mathrm{B}$ in alginate gel is different from that for diffusion of sirolimus in vivo. ${ }^{26,28}$ Since an actual artery has more complicated structures and an anisotropic structure, subsequent rhodamine $\mathrm{B}$ depositions in alginate gel could be different from consequent deposition of sirolimus in vivo; however, the purpose of this in vitro study is to conform that a drug released from a stent with an nonuniform coating uniformly spreads to the luminal side of the stented artery by visualizing drug diffusion using a fluorescent dye as a model drug and comparing the diffusion direction of the drug released from the stent with CFC. Therefore, the subsequent sirolimus deposition in the stented artery, which is influenced by the structure of the artery and binding sites for the sirolimus in the artery, is out of the scope of this in vitro study.

As for the subsequent sirolimus distribution for the nonuniform coating and their consequent clinical impacts, we prepared sirolimus-eluting stents with $\mathrm{AGC}$ and $\mathrm{CFC}$ and investigated the biological responses to AGC in in vivo, the endothelialization on the luminal surface of the stent, and the uniformity of the neointima thickness by implanting the stents in the iliac artery of the rabbits for 2 weeks.

\section{Materials and methods}

\section{Drug diffusion studies in the alginate artery model}

\section{Preparation of rhodamine B-coated stents}

Rhodamine B-coated stents with AGC (AGC stents) were prepared by coating a mixture $(1: 5)$ of rhodamine $\mathrm{B}$ (EMD Millipore, Billerica, MA, USA) and poly(DL-lactide-cocaprolactone) polymer (PDLLCL; Evonik, Tokyo, Japan) dissolved in acetone to the abluminal side of a BMS (Kaname ${ }^{\mathrm{TM}}$ Cobalt Chrome Stent, $3 \mathrm{~mm}$ inner diameter and $18 \mathrm{~mm}$ length; Terumo, Tokyo, Japan) using an automatic dispensing machine ${ }^{21}(\sim 15 \mu \mathrm{g}$ of rhodamine B per stent). Similarly, rhodamine B-coated stents with CFC were prepared by coating the mixture of rhodamine $\mathrm{B}$ and the PDLLCL polymer to the full surface of the BMS using a spray-coating machine (Valvemate 7040; Nordson EFD, East Providence, RI, USA; $\sim 15 \mu \mathrm{g}$ of rhodamine B per stent). Both rhodamine B-coated stents were mounted on stent delivery balloon catheters.

\section{Preparation of alginate gel and alginate artery model} The alginate gel was prepared by mixing 3\% sodium alginate solution with $2.5 \%$ calcium sulfate $/ 0.18 \%$ sodium tripolyphosphate solution (50:4 ratio) ${ }^{26}$ after which it was poured into a plastic tube that was closed at one end with an $8 \mathrm{~mm}$ inner diameter and $550 \mathrm{~mm}$ length. For longitudinal drug distribution study, a stainless steel rod (outer diameter $3.0 \mathrm{~mm}$ ) was inserted into the center of the plastic tube filled with the alginate gel and withdrawn from the alginate gel to create an artery model with a $3 \mathrm{~mm}$ inner diameter.

\section{Drug diffusion studies \\ Circumferential drug diffusion}

Both rhodamine B-coated stents, mounted on a delivery balloon catheter, were expanded by ballooning and cut circumferentially at the link part of the stent with a micro-nipper using a microscope. The cut stent was embedded in the alginate gel by pushing it into the gel for observation of the stent strut cross-section. The cross section of the stent strut was observed with a charge-coupled device (CCD) camera connected to a fluorescence microscope equipped with a U-MWIG filter cube (Olympus Corporation, Tokyo, Japan). The images were taken 1, 2, 3, 4, and 5 minutes after embedding the stent in the artery model.

\section{Longitudinal drug diffusion of the AGC stent}

Three AGC stents and three alginate artery models were prepared. The AGC stents were deployed in the artery model in which saline was perfused at $35 \mathrm{~mL} / \mathrm{min}$ using a peristaltic pump to simulate blood flow in the coronary artery. In all, 1,3 , and 5 minutes later, the flow was stopped, the artery models were taken out from the plastic tube and cut longitudinally in half, and the deployed stent was taken out from the artery model. The inner surface of the artery model was observed with a CCD camera connected to a fluorescence microscope equipped with a U-MWIG filter cube. Images of the inner surface of the artery model were obtained for image processing.

\section{Image processing}

The images taken earlier were imported to a computer and converted into monochrome images. The monochrome images were histogram equalized and converted to color images, and values for red, green, and blue were assigned on a scale of 0-255 using the imaging toolbox of MATLAB version 7.14.0.739 (R2012a; MathWorks, Natick, MA, USA).

\section{Biological responses for AGC}

Preparation of sirolimus-coated stents

Sirolimus-coated stents with AGC were prepared by coating a mixture (1:1) of sirolimus (Sigma-Aldrich Co., St Louis, MO, 
USA) and the PDLLCL polymer dissolved in acetone to the abluminal side of the BMS (3 mm inner diameter and $18 \mathrm{~mm}$ length) using the automatic dispensing machine ( $\sim 60 \mu \mathrm{g}$ of sirolimus per stent, dose has been confirmed as an effective dose). Similarly, sirolimus-coated stents with CFC were prepared by coating the mixture of sirolimus and polymer to the whole surface of the BMS using a spray-coating machine ( $\sim 60 \mu \mathrm{g}$ of sirolimus per stent). Both sirolimus-coated stents were mounted on stent delivery catheters and sterilized by electronic beam sterilization.

To confirm the ability of AGC for the coating integrity, the stents were overexpanded to an inner diameter of $6.0 \mathrm{~mm}$ using the kissing balloon technique for a bifurcation lesion, ${ }^{33}$ and the stent surface was observed by scanning electron microscope (SEM) S-3400N (Hitachi Ltd., Tokyo, Japan).

\section{Rabbit iliac artery implants and histopathological evaluation}

To investigate the biological responses to AGC, particularly in terms of endothelialization and neointima formation, the AGC stents and CFC stents as the control were implanted in a rabbit iliac artery model for 2 weeks, after which scanning electron microscopy study of the luminal surface and histopathological analysis were performed, as shown in Table 1. All experimental procedures involving the use of animals were approved by the Animal Ethics Committee of Terumo Corporation R\&D Center (approval number 100257). The implantation procedures are briefly described later.

Japanese white rabbits (body weight $2.5-3.0 \mathrm{~kg}, \mathrm{n}=4$ ) were anesthetized by an intravenous injection of dexmedetomidine hydrochloride $0.2 \mathrm{mg} / \mathrm{kg}$ and midazolam hydrochloride $0.5 \mathrm{mg} / \mathrm{kg}$. The right carotid artery was surgically exposed, and the distal artery was ligated. A 5 Fr introducer sheath was placed in the artery. Heparin $150 \mathrm{IU} / \mathrm{kg}$ was administered intra-arterially via the sheath. A guiding catheter and a guide wire were placed into the sheath and advanced into the iliac artery under fluoroscopic guidance. The stent delivery system was advanced into the iliac artery

Table I Study design for biological responses sirolimus-coated stents in rabbit iliac arteries

\begin{tabular}{llllll}
\hline \multirow{2}{*}{$\begin{array}{l}\text { Inimal } \\
\text { ID }\end{array}$} & \multicolumn{2}{l}{ Left iliac artery } & & Right iliac artery \\
\cline { 2 - 3 } \cline { 5 - 6 } & Stent & Assessment & & Stent & Assessment \\
\hline I & CFC stent & Histopathology & CFC stent & SEM \\
2 & AGC stent & Histopathology & AGC stent & SEM \\
3 & CFC stent & Histopathology & CFC stent & Histopathology \\
4 & AGC stent & Histopathology & AGC stent & Histopathology \\
\hline
\end{tabular}

Abbreviations: AGC, abluminal-gradient coating; CFC, conventional full-surface coating; SEM, scanning electron microscope. on the guide wire, and the stent was deployed into the iliac artery. Two identical stents were implanted in the right and left iliac arteries each. Following the procedure, the catheters were removed and the proximal artery was ligated. Lastly, the fascia and subcutaneous layers were closed, and enrofloxacin $5 \mathrm{mg} / \mathrm{kg}$ was injected intramuscularly. The animals were returned to the vivarium and allowed to recover.

Two weeks after the implantation, the animals were euthanized by exsanguination under anesthesia. Following the study design as shown in Table 1, the right iliac arteries (animal ID 1 and 2), implanted with the CFC or AGC stents, respectively, were perfused with $500 \mathrm{~mL}$ of saline for observation using SEM. Subsequently, cooled $2 \%$ paraformaldehyde/1.25\% glutaraldehyde in $0.1 \mathrm{M}$ PBS was infused into the iliac arteries for fixation. The stented arteries were harvested and stored in 2\% paraformaldehyde/1.25\% glutaraldehyde in $0.1 \mathrm{M}$ PBS, which were longitudinally half-cut by micro scissors. Next, the stented arteries were dehydrated through an ascending series of ethanol concentrations, ending in $100 \%$ ethanol, and immersed in $t$-butanol before being freeze-dried. The luminal surface of the freezedried stented arteries was observed by SEM. The endothelial coverage was measured by using an image analyzer.

For pathological examination, the left iliac arteries (animal ID 1 and 2) and both the iliac arteries (animal ID 3 and 4) implanted with AGC or CFC stents were perfused with $500 \mathrm{~mL}$ of saline, after which $10 \%$ formaldehyde in PBS was infused into the iliac arteries for fixation. The stented arteries were harvested and embedded in acrylic resin Technovit 8100 (Heraeus Kulzer, Wehrheim, Germany), which were sectioned into three blocks, namely, the proximal, middle, and distal blocks. Further, each block was sectioned into $5 \mu \mathrm{m}$ slices, and the slices were mounted to microscope slides and hematoxylin and eosin staining was performed. A microscope, equipped with a CCD camera connected to a personal computer, was used to capture high-resolution images of the processed cross-sections on the slides. The neointima thicknesses on the strut of the middle sections were measured by a length-calibrated image analyzer. The injury-, inflammation-, endothelialization-, fibrin-, and SMCcontent scores were evaluated. ${ }^{34-36}$ Moreover, the leukocytes adhered to the endothelium and infiltrated in the neointima were counted.

In addition to the histopathological analysis, to investigate the impact of the gradient coating on the efficacy of DES, three sets of four serial cross-sectional images of the AGCstented artery, in which both struts coated and not coated with the mixture of sirolimus and the polymer were included in two 
images each, were prepared, and the neointima thicknesses at each strut site were measured by a length-calibrated image analyzer.

Statistical analysis for the histopathological scores, leukocyte counts, and neointima thicknesses was performed using SigmaPlot 13 (Systat Software, San Jose, CA, USA). Differences among the histopathological scores were analyzed using the Mann-Whitney rank sum test. The leukocyte counts and the neointima thicknesses were first checked for normal distribution using the Shapiro-Wilk test. Furthermore, the leukocyte counts and the neointima thicknesses were compared by the Student's $t$-test and the Mann-Whitney rank sum test, respectively. $P$-values $<0.05$ were considered significant.

\section{Results \\ Preparation of DESs}

As shown in Figure 1, the rhodamine B-eluting stents and the sirolimus-eluting stents with the AGC and CFC were prepared by coating a mixture of rhodamine $\mathrm{B}(1: 5)$ or sirolimus $(1: 1)$ with the polymer. Owing to low solubility of rhodamine B in acetone (a common solvent for the polymer and rhodamine B) and poor film-forming properties of thin coating formulation prepared with rhodamine $\mathrm{B} /$ polymer ratio of $1: 1$, a ratio of 1:5 had to be selected for the rhodamine B-eluting stent to avoid forming a inhomogeneous coating layer that might influence the drug diffusion.

The AGC stent was prepared leaving the abluminal surface of the curves and the link parts of the stent free from the rhodamine B or sirolimus coating by gradually decreasing the coating layer thickness (the maximum coating thickness of the sirolimus-eluting stent $15 \mu \mathrm{m})$ toward the hinge area. In other words, the curves $(\sim 120 \mu \mathrm{m}$ length along the curve) and links ( $\sim 200 \mu \mathrm{m}$ length between the diagonal struts) where a coating layer was stressed by stent expansion with a risk of coating delamination in the case of CFC were not coated with a mixture of either rhodamine $\mathrm{B}$ or sirolimus and the polymer. By weighing of the stent before and after the coating, it was confirmed that the amount of rhodamine $\mathrm{B}$ or sirolimus on the AGC stent was the same as that on the CFC stent. It was also confirmed that there was no delamination of the coating layer of AGC, even after excess stent expansion, as shown in the panels of Figure 1.

\section{Drug diffusion studies on artery model}

For the circumferential drug diffusion, the cross-sectional raw fluorescence images of the artery model implanted with
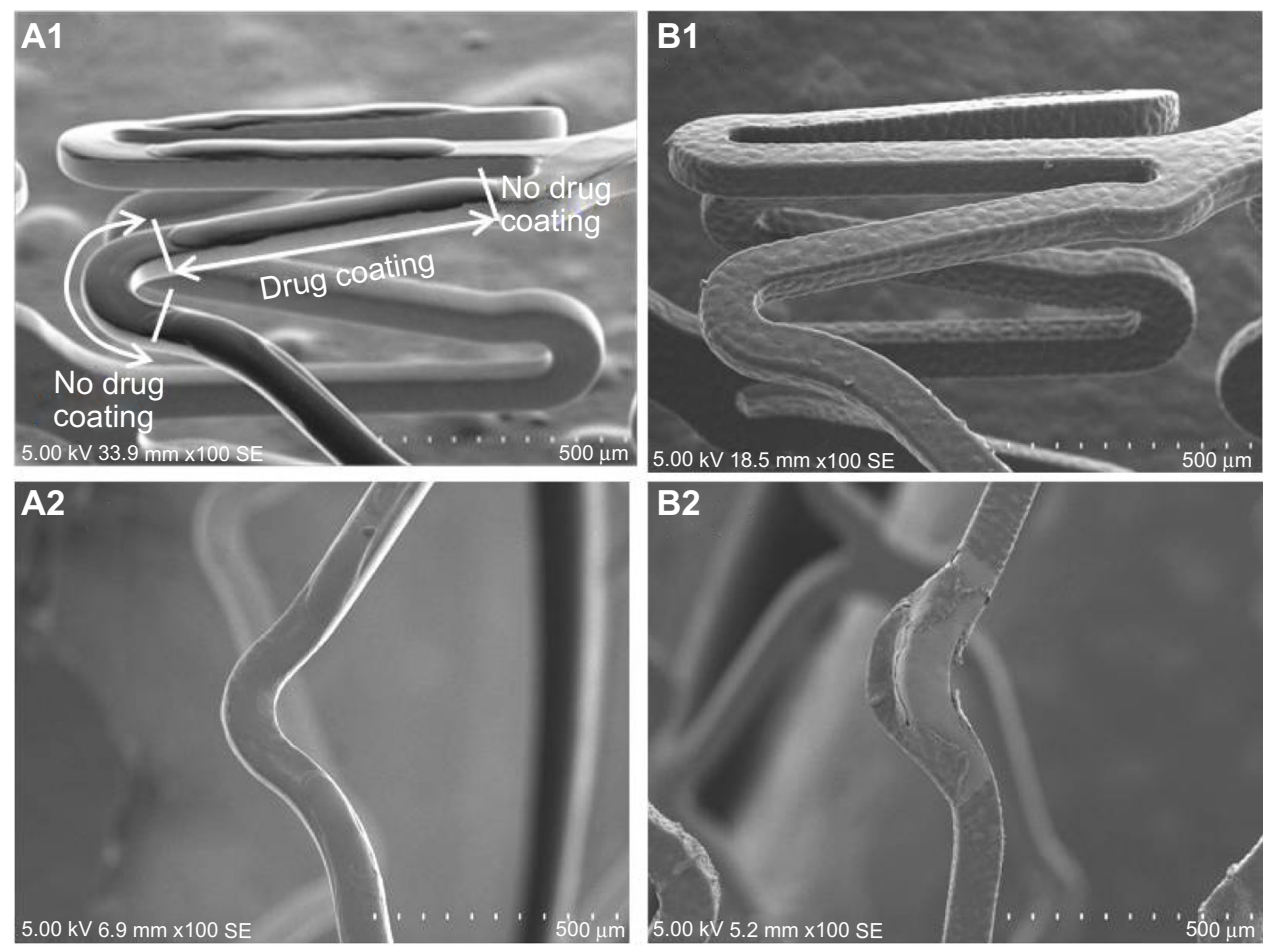

Figure I Scanning electron microscope images of sirolimus-coated stents with AGC stent (A) and CFC stent (B).

Notes: AGC leaves the abluminal surface of the curves and links parts of the stent free from the drug and biodegradable polymer coating by gradually decreasing the coating layer thickness (from $\sim 15 \mu \mathrm{m}$ to $0 \mu \mathrm{m}$ ) toward the hinge area. The stents were expanded to an inner diameter of $3.0 \mathrm{~mm}(\mathbf{A l}$ and $\mathbf{B} \mathbf{I}$ ). The stents were overexpanded to an inner diameter of $6.0 \mathrm{~mm}$ using the kissing balloon technique for a bifurcation lesion (A2 and B2).

Abbreviations: AGC, abluminal-gradient coating; CFC, conventional full-surface coating. 
the rhodamine B-coated stents taken 1, 2, 3, 4, and 5 minutes after stenting the artery model were used, and their corresponding processed images were converted to color images and were assigned values for red, green, and blue on a scale of 0-255, as shown in Figure 2. In the AGC stent, the fluorescence of rhodamine $\mathrm{B}$ was predominantly diffused into the artery model and was spread along the artery wall over time; however, even though the lumen of the stented artery was filled with alginate gel, where rhodamine B can diffuse into, it was limited to the abluminal side even after 5 minutes. On the other hand, in the CFC stent, the fluorescence of rhodamine $\mathrm{B}$ had spread to both the luminal and abluminal sides 1 minute after stenting, and the area had gradually expanded over time.

For longitudinal drug diffusion of the AGC stent, raw images of the inner surface of the stented arteries, taken 1,3 , and 5 minutes after stenting in the artery model and their corresponding processed images are shown in Figure 3. From the raw images, no diffusion of rhodamine $B$ on the surface could be identified. However, the processed images clearly indicated that, over time, the fluorescence of the rhodamine B gradually expanded longitudinally from near the stent strut to the area near the curve without rhodamine B coating and increased near the distal side of the stent strut and in the inside of the curves.

\section{Biological responses for abluminal coating}

Although the animals implanted with the AGC stent remained in good health condition until the end of the experiment, one animal implanted with the CFC stent had to be euthanized on day 12 because of necrosis of toe tips of the left leg, which was considered likely related to the stent implantation. A necropsy for the animal revealed that the stented left iliac artery was occluded with thrombosis. Thrombosis was also observed in two other iliac arteries implanted with the CFC stent, although these were not occluded. Furthermore, thrombosis was observed in one of the iliac arteries implanted with the AGC stent.

SEM images of the luminal surface for the stented iliac artery are shown in Figure 4. The luminal surface of the iliac artery implanted with the AGC stent was completely covered with spindle-shaped endothelial-like cells (endothelial coverage: $97 \%, n=1$ ), whereas the luminal surface of the iliac artery implanted with the CFC stent was only partially covered with
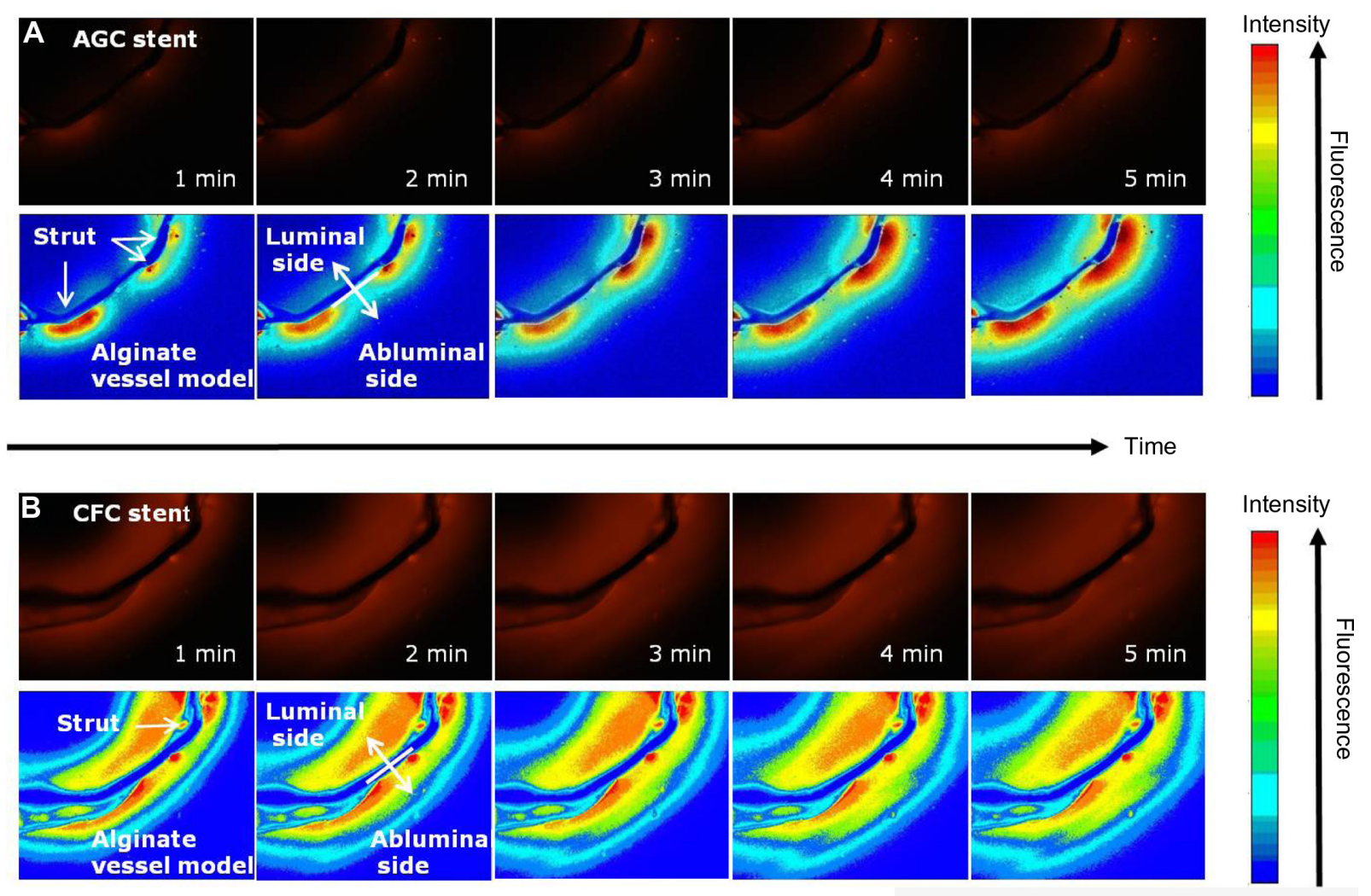

Figure 2 Circumferential drug distribution of the alginate artery model implanted with the rhodamine B AGC stent (A) and the rhodamine B CFC stent (B).

Notes: Cross-sectional raw fluorescence images of the stented artery model, taken at I, 2, 3, 4, and 5 minutes after stenting, and their corresponding processed images are shown.

Abbreviations: AGC, abluminal-gradient coating; CFC, conventional full-surface coating; min, minute. 


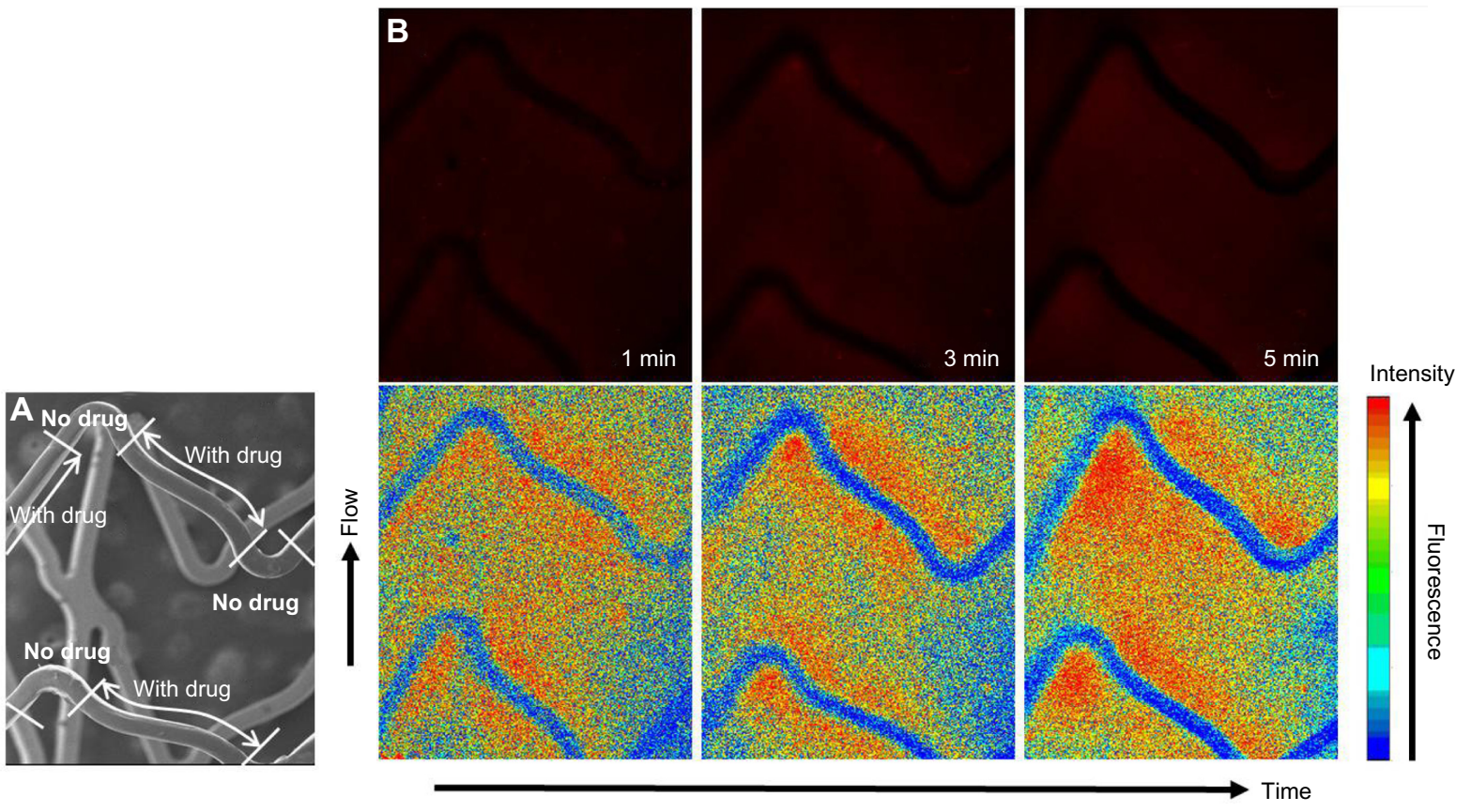

Figure 3 Longitudinal drug distribution of the alginate artery model implanted with the rhodamine B AGC stent.

Notes: (A) A scanning electron microscope image of the region of interest for the AGC stent. (B) The raw fluorescence images of the stented artery model, taken I, 3, and 5 min after stenting, and their corresponding processed images. Because of the shallow depth of focus in the microscopy and the rounded surface of the stented artery, the fluorescence is hardly detected on both sides of the images. Thus, both sides of the images should be omitted from the region of interest.

Abbreviations: AGC, abluminal-gradient coating; min, minute.

endothelial-like cells (endothelial coverage: $73 \%, \mathrm{n}=1$ ), and platelets and leukocytes aggregated with fibrin were observed on the uncovered stent strut.

Pathological examination for the cross-sectional images revealed that the neointima thickness on the strut in the CFC stent group was significantly less than that in the AGC-stent group, and both leukocytes adhered to the luminal surface and infiltrated in the neointima were more commonly observed in the arteries implanted with the CFC stent (Table 2). In addition, the SMC content score for the CFC stent group was significantly lower compared to that for the AGC-stent group, whereas the injury, inflammation, endothelialization, and fibrin scores did not significantly differ between the groups. Moreover, in the AGC stent, the neointima thickness did

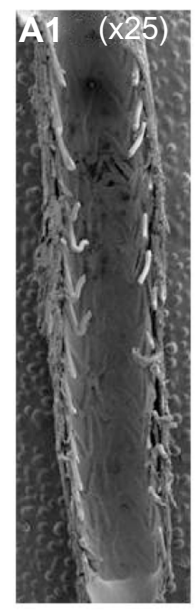

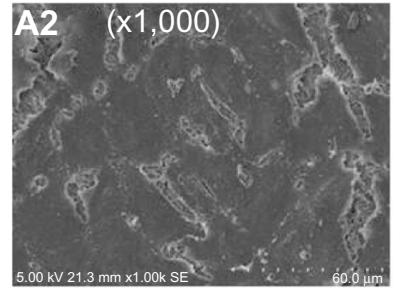

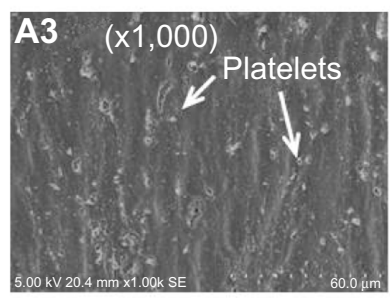

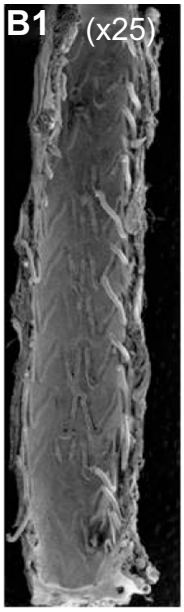
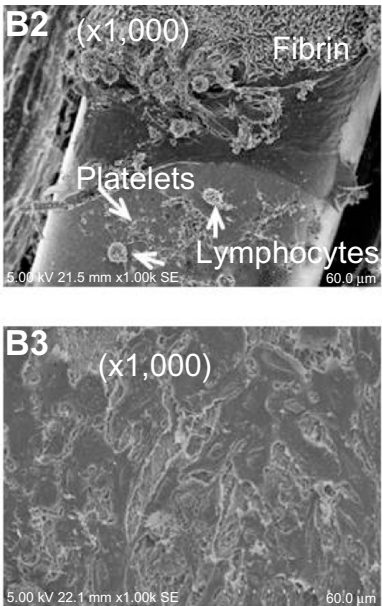

Figure 4 Scanning electron microscope images of the luminal surface of the iliac artery.

Notes: (A) The luminal surface of the iliac arteries implanted with the sirolimus AGC stent for 2 weeks was covered with endothelial-like cells (AI); the stent strut was covered with fibrous connective tissue and endothelial cells (A2); and spindle-shaped endothelial-like cells oriented in the direction of blood flow (from top to down) were observed (A3). (B) The luminal surface of the iliac artery implanted with the sirolimus CFC stent (B) for 2 weeks was only partially covered with endothelial-like cells (B I); areas not covered by fibrous connective tissue were generally located on the stent strut, where adhered platelets and lymphocytes were observed (B2); fibrin and aggregated platelets were seen even on the endothelium (B3).

Abbreviations: AGC, abluminal-gradient coating; CFC, conventional full-surface coating. 
Table 2 Results of the pathological examination of iliac arteries implanted for 2 weeks with the sirolimus AGC and CFC stents (mean \pm SD)

\begin{tabular}{llll}
\hline Biological responses & AGC stent & CFC stent & $P$-value \\
\hline $\begin{array}{l}\text { Neointima thickness on the } \\
\text { strut }(\mu \mathrm{m})\end{array}$ & $10.3 \pm 4.2$ & $8.2 \pm 4.6$ & $<0.05$ \\
$\quad \begin{array}{lll}\text { Injury score } \\
\text { Inflammation score }\end{array}$ & $0.0 \pm 0.1$ & $0.0 \pm 0.0$ & $\mathrm{NS}$ \\
Endothelialization score & $0.3 \pm 0.2$ & $0.4 \pm 0.2$ & $\mathrm{NS}$ \\
$\begin{array}{l}\text { Fibrin content score } \\
\text { SMC content score }\end{array}$ & $1.0 \pm 0.0$ & $2.7 \pm 0.5$ & $\mathrm{NS}$ \\
$\begin{array}{l}\text { Leukocytes adhered to the } \\
\text { endothelium (cells/section) }\end{array}$ & $3.7 \pm 4.2$ & $1.4 \pm 0.7$ & $\mathrm{NS}$ \\
$\begin{array}{l}\text { Leukocytes infiltrated in the } \\
\text { neointima (cells/section) }\end{array}$ & $4.6 \pm 1.9$ & $1.9 \pm 0.3$ & $<0.05$ \\
\hline
\end{tabular}

Abbreviations: AGC, abluminal-gradient coating; CFC, conventional full-surface coating; SD, standard deviation; NS, nonsignificant; SMC, smooth muscle cell.

not significantly differ between the struts with and without the drug coating, as shown in Figure 5, and no impact of the gradient coating on neointima formation was observed.

\section{Discussion}

Compared with the simplified alginate artery model used in this in vitro drug diffusion study, actual arteries have a more complicated hydrophobic three-layer structure (intima, media, and adventitia), and compared with rhodamine B used in the in vitro drug diffusion study, sirolimus is more hydrophobic. Moreover, the in vitro release rate of rhodamine B from the polymer matrix is higher than the in vivo release rate of sirolimus because of higher solubility of rhodamine $B$ into the medium. Therefore, the timescale of the drug release and the drug diffusion in this in vitro drug diffusion study does not exactly match the timescale of actual drug release and drug diffusion in vivo. However, it seems reasonable to suppose that the diffusion direction of rhodamine B in the artery model simulates the diffusion direction of sirolimus in the stented artery because it is assumed that the transport of a drug released from a DES in the arterial wall is principally governed by diffusion and convection-mediated transport, and the three-layer structure in an artery is not a significant barrier to the diffusion of sirolimus. ${ }^{30}$

In the circumferential drug distribution study, fluorescence of rhodamine B released from the AGC stent was found to spread along the artery wall but was limited to the abluminal side only, whereas fluorescence of rhodamine $\mathrm{B}$ released from the CFC stent conversely, quickly spread not only to the
A

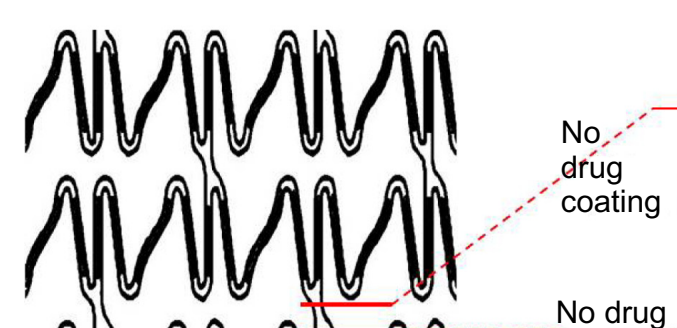

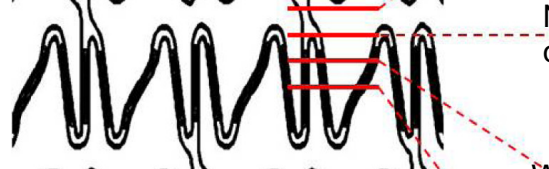

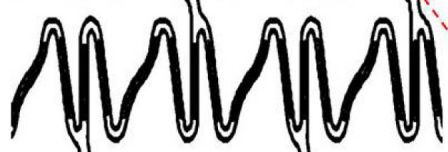

AMMMAI

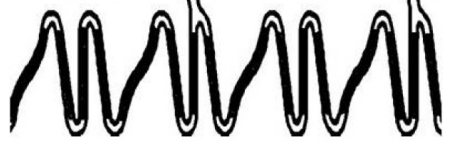

B

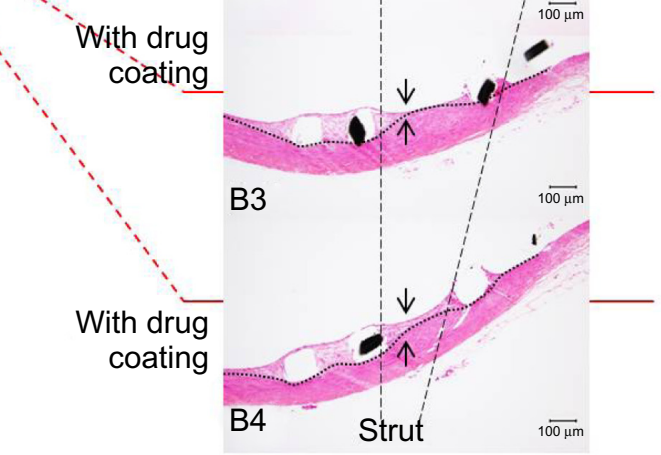

C

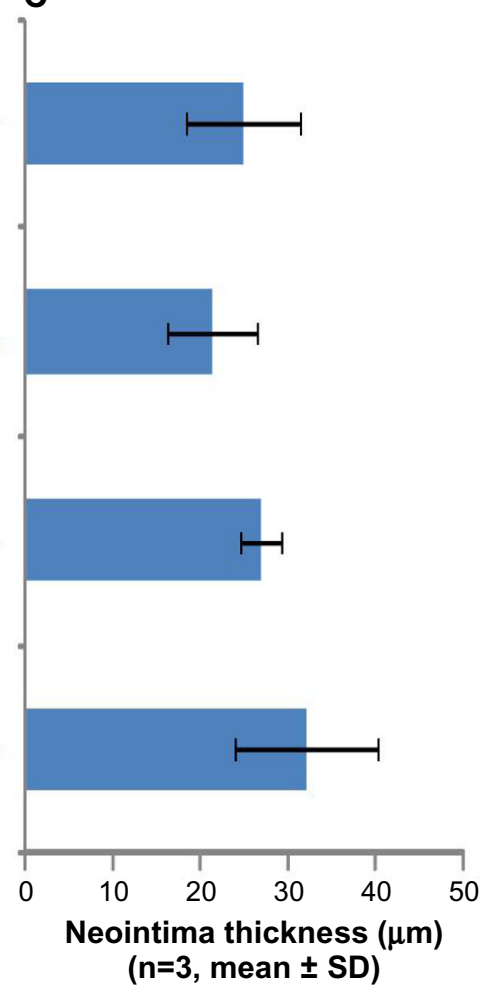

Figure 5 Neointima thicknesses at the struts, with or without the drug coating, of the iliac artery implanted with a sirolimus AGC stent for 2 weeks. Notes: (A) An illustration of the AGC-coated stent (developed view). The areas of the strut not filled with black do not have the drug coating. (B) Typical serial crosssectional images of struts without (B I and B2) and with the drug coating (B3 and B4). (C) The neointima thicknesses were measured for three different struts and averaged. Abbreviations: AGC, abluminal-gradient coating, IEL, internal elastic lamina; SD, standard deviation. 
abluminal side but also to the luminal side. Thus, the results of this study support the hypothesis that the abluminal coating enhances reendothelialization on the luminal surface of the stent by preventing exposure to the antiproliferative drugs, as it is likely that an antiproliferative drug released from the CFC stent spreads not only to the abluminal side but also to the luminal side where endothelial cell proliferation is needed. Consequently, this results in endothelialization being delayed and in an impaired endothelium, even after endothelialization is completed. However, it can also be speculated that the drug diffused into the lumen of the CFC-stented artery would be washed out by the blood flow in vivo, resulting in the drug not accumulating at the luminal side of the CFC stent and the endothelium on the luminal surface not being affected by the drug diffused into the lumen side. However, the results of our animal study showed that the neointima thickness on the strut of the sirolimus CFC-stented artery was significantly less compared to that of the sirolimus AGC-stented artery, and the endothelial coverage of the CFC-stented artery was $73 \%$, whereas the endothelial coverage of the AGC-stented artery was 97\%. Moreover, in the CFC-stented artery, leukocytes were found to adhere to the luminal surface and leukocytes had infiltrated in the neointima, both of which are indicators of a dysfunctional endothelium. Furthermore, while thrombosis was observed in vivo in two of the three CFC stents, it was observed in only one of the three AGC stents. Taken together, the endothelial cells of the sirolimus CFC-stented artery were affected by sirolimus ${ }^{37-39}$ compared to the endothelial cells of the sirolimus AGC-stented artery. These findings indicate that the abluminal coating, which intends to enhance reendothelialization, is an appropriate approach for minimizing stent thrombosis.

Balakrishnan et al showed by mathematical modeling that abluminally coated drugs account for $<50 \%$ of the drugs that end up in the arterial wall because of blood flow and questioned the central precept for the current DES development, namely, that abluminal coating alone can ensure optimal delivery of the drug to the wall while minimizing drug loss into the bloodstream. ${ }^{31}$ The authors found that the bioavailability of drugs released from an abluminal coating was $<50 \%$ in vivo; however, this is not to deny the desirable effect of the abluminal coating on endothelialization/endothelium function and the central precept for the current DES development, as the main issue of the current DES is the safety, ie, endothelium dysfunction and consequent stent thrombosis, and not the efficacy related to the bioavailability of a drug.

In the present longitudinal drug diffusion study, it was confirmed that rhodamine B diffuses longitudinally from the AGC-stent strut to the area around the curves without rhodamine B coating. Interestingly, the fluorescence intensity of rhodamine B increased around the distal side of the stent strut and inside of the curves over time. Since the fluorescence zone was asymmetric with respect to the strut and corresponded to the downstream area of the strut with the coating layer, one explanation for these results may be that the longitudinal diffusion of rhodamine $\mathrm{B}$ released from the AGC stent was governed not only by simple diffusion but also by flow. As reported by Balakrishnan et al, ${ }^{31,32}$ recirculation of flow at the distal side of the stent strut can result in rhodamine $\mathrm{B}$, once dissolved in the flow medium, being deposited on the distal side of the strut. However, in an artery implanted with a DES incorporated with sirolimus, the contribution of blood flow to the drug distribution should not be extensively compared to simple diffusion, as sirolimus is a hydrophobic drug and is extensively partitioned into the tissue $^{32}$ and only minimally dissolve in the blood..$^{40}$

From the present longitudinal drug diffusion study, it can be assumed that sirolimus released from the AGC stent diffuses longitudinally, at least by diffusion and convection, consequently resulting in the sirolimus being diffused into the whole stented artery even by AGC stents with nonuniform coating. Accordingly, in this biological response study, the difference in neointima thickness along the stent strut of the sirolimus AGC stent was not significant between the struts with and without the drug-coating layer. Moreover, in a previous clinical study, the Ultimaster ${ }^{\mathrm{TM}}$ sirolimus-eluting stent applied with the AGC technology showed low in-stent and in-segment restenosis, revealing a very thin and homogenous layer of neointima covering the stent struts 6 months after stent implantation. ${ }^{41,42}$

These results support our assumption that the sirolimus initially diffuses along the stent struts but finally diffuses to the abluminal side of the stented artery uniformly even by AGC stents with nonuniform coating. This, however, does not say that there is no anisotropic drug diffusion in the artery and no drug concentration gradient in the artery implanted with DES. We do not deny the presence of anisotropic arterial drug diffusion ${ }^{28}$ and drug concentration gradient. ${ }^{31,32}$ However, it seems that because of the higher circumferential diffusion than the transmural diffusion, the drug gradient in the circumferential direction would be reduced and more uniformly loaded drug layers can be produced, which can be beneficial in reducing in-stent restenosis uniformly after DES implantation. ${ }^{28}$ Also, it can be beneficial in enhancing reendothelialization together with the abluminal coating, because the lower transmural diffusion would attenuate the 
increase in the drug concentration in the endothelial layer, which is regenerated on the newly formed intima layer.

To understand the results of this study and the clinical studies, in addition to the view of the drug diffusion in the artery wall, we must need the more important view that sirolimus has a wide therapeutic window (from $10^{-10} \mathrm{M}$ to $\left.10^{-8} \mathrm{M}\right) .^{43,44}$ Because of the wide therapeutic window, sirolimus can reduce the neointimal formation uniformly regardless of somewhat drug concentration gradient in the stented artery. From this viewpoint, one may say that sirolimus is a suitable drug for DES used to treat the whole stented artery in spite of the topical administration from the stent strut. ${ }^{19,45}$

\section{Conclusion}

In summary, in this study, by observing the circumferential and longitudinal drug diffusion and by assessing the biological responses to the AGC stent implanted in the rabbit iliac artery, the validity of the designed nonuniform coating was supported, and our results indicate that the abluminal coating is an appropriate approach for minimizing stent thrombosis without impacting the efficacy of DES. The AGC, designed by taking into consideration the drug diffusion in the artery wall, drug solubility in the blood, therapeutic window of the drug, and stress in the coating layer, is an appropriate approach for achieving a safer DES and for reducing the risks of stent thrombosis and distal embolization due to a cracked coating layer. However, the number of experiments is limited and a larger study should be conducted to reach a statistically significant conclusion.

\section{Disclosure}

All authors are employees of Terumo Corporation. The authors report no other conflicts of interest in this work.

\section{References}

1. Grines CL, Bonow RO, Casey DE Jr, et al; American Heart Association; American College of Cardiology; Society for Cardiovascular Angiography and Interventions; American College of Surgeons; American Dental Association; American College of Physicians. Prevention of premature discontinuation of dual antiplatelet therapy in patients with coronary artery stents. Circulation. 2007;115:813-818.

2. Riede FN, Pfisterer M, Jeger R. Long-term safety of drug-eluting stents. Expert Rev Cardiovasc Ther. 2013;11(10):1359-1378.

3. Moussa ID, Colombo A. Antiplatelet therapy discontinuation following drug-eluting stent placement: dangers, reasons, and management recommendations. Catheter Cardiovasc Interv. 2009;74(7): 1047-1054.

4. Warren J, Baber U, Mehran R. Antiplatelet therapy after drug-eluting stent implantation. J Cardiol. 2015;65(2):98-104.

5. Stefanini GG, Taniwaki M, Windecker S. Coronary stents: novel developments. Heart. 2014;100(13):1051-1061.

6. Pilgrim T, Windecker S. Drug-eluting stent technology: progress beyond the polymer. Eur Heart J. 2014;35(30):1991-1995.
7. Lüscher TF, Steffel J, Eberli FR, et al. Drug-eluting stent and coronary thrombosis: biological mechanisms and clinical implications. Circulation. 2007;115(8):1051-1058.

8. Sudir K, Hermiller JB, Ferguson JM, Simonton CA. Risk factors for coronary drug-eluting stent thrombosis: influence of procedural, patient, lesion, and stent related factors and dual antiplatelet therapy. ISRN Cardiol. 2013;2013:748736.

9. Attizzani GF, Capodanno D, Ohno Y, Tamburino C. Mechanisms, pathophysiology, and clinical aspects of incomplete stent apposition. J Am Coll Cardiol. 2014;63(14):1355-1367.

10. Cook S, Wenaweser P, Togni M, et al. Incomplete stent apposition and very late stent thrombosis after drug-eluting stent implantation. Circulation. 2007;115(18):2426-2434.

11. Nakazawa G, Finn AV, Kolodgie FD, Virmani R. A review of current devices and a look at new technology: drug-eluting stents. Expert Rev Med Devices. 2009;6(1):33-42.

12. Joner M, Finn AV, Farb A, et al. Pathology of drug-eluting stents in humans: delayed healing and late thrombotic risk. J Am Coll Cardiol. 2006;48(1):193-202.

13. Byrne RA, Joner M, Kastrati A. Polymer coatings and delayed arterial healing following drug-eluting stent implantation. Minerva Cardioangiol. 2009;57(5):567-584.

14. Zhang H, Deng W, Wang X, Wang S, Ge J, Toft E. Solely abluminal drug release from coronary stents could possibly improve reendothelialization. Catheter Cardiovasc Interv. Epub 2013 Dec 10.

15. Danzi GB, Chevalier B, Ostojic M, Hamilos M, Wijins W. Nobori ${ }^{\mathrm{TM}}$ drug eluting stent system: clinical evidence update. Minerva Cardioangiol. 2010;58(5):599-610.

16. Moretti C, Lolli V, Perona G, et al. Cre $8^{\mathrm{TM}}$ coronary stent: preclinical in vivo assessment of a new generation polymer-free DES with Amphilimus $^{\mathrm{TM}}$ formulation. EuroIntervention. 2012;7(9):1087-1094.

17. Serruys PW, Farooq V, Kalesan B, et al. Improved safety and reduction in stent thrombosis associated with biodegradable polymer-based biolimuseluting stents versus durable polymer-based sirolimus-eluting stents in patients with coronary artery disease: final 5-year report of the LEADERS (limus eluted from a durable versus ERodable stent coating) randomized, noninferiority trial. JACC Cardiovasc Interv. 2013;6(8):777-789.

18. Wilson GJ, Huibregtse BA, Pennington DE, Dawkins KD. Comparison of the SYNERGY with the PROMUS (XIENCE V) and bare metal and polymer-only element control stents in porcine coronary arteries. EuroIntervention. 2012;8(2):250-257.

19. Guagliumi G, Sirbu V, Musumeci G, et al. Strut coverage and vessel wall response to a new-generation paclitaxel-eluting stent with an ultrathin biodegradable abluminal polymer: optical coherence tomography drug-eluting stent investigation (OCTDESI). Circ Cardiovasc Interv. 2010;3(4):367-375.

20. Meredith IT, Verheye S, Dubois CL, et al. Primary endpoint results of the EVOLVE trial: a randomized evaluation of a novel bioabsorbable polymer-coated, everolimus-eluting coronary stent. J Am Coll Cardiol. 2012;59(15):1362-1370.

21. MARUYAMA KAZUHIRO [JP]; SAITO NOBORU [JP]; GOTO HIROKI [JP] +, inventor; Maruyama K, Saito N, Goto H, assignee. Stent. World patent WO2011040218 (A1). 2010 Sept 13.

22. Basalus MW, Tandjung K, van Westen T, et al. Scanning electron microscopic assessment of coating irregularities and their precursors in unexpanded durable polymer-based drug-eluting stents. Catheter Cardiovasc Interv. 2012;79(4):644-653.

23. Basalus MW, van Houwelingen $\mathrm{KG}$, Ankone $\mathrm{M}$, de $\mathrm{Man} \mathrm{FH}$, von Birgelen C. Scanning electron microscopic assessment of the biodegradable coating on expanded biolimus-eluting stents. EuroIntervention. 2009;5(4):505-510.

24. Lee S, Lee CW, Kim CS. FEA study on the stress distributions in the polymer coatings of cardiovascular drug-eluting stent medical devices. Ann Biomed Eng. 2014;42(9):1952-1965.

25. Hopkins CG, McHugh PE, McGarry JP. Computational investigation of the delamination of polymer coatings during stent deployment. Ann Biomed Eng. 2010;38(7):2263-2273. 
26. Seidlitz A, Nagel S, Semmling B, et al. Examination of drug release and distribution from drug-eluting stents with a vessel-simulating flowthrough cell. Eur J Pharm Biopharm. 2011;78(1):36-48.

27. Semmling B, Nagel S, Sternberg K, Weitschies W, Seidlitz A. Development of hydrophobized alginate hydrogels for the vessel-simulating flow-through cell and their usage for biorelevant drug-eluting stent testing. AAPS PharmSciTech. 2013;14(3):1209-1218.

28. Zhu X, Pack DW, Braatz RD. Modelling intravascular delivery from drug-eluting stents with biodurable coating: investigation of anisotropic vascular drug diffusivity and arterial drug distribution. Comput Methods Biomech Biomed Engin. 2014;17(3):187-198.

29. O’Brien CC, Finch CH, Barber TJ, Martens P, Simmons A. Analysis of drug distribution from a simulated drug-eluting stent strut using an in vitro framework. Ann Biomed Eng. 2012;40(12):2687-2696.

30. Hwang CW, Wu D, Edelman ER. Impact of transport and drug properties on the local pharmacology of drug-eluting stents. Int J Cardiovasc Intervent. 2003;5(1):7-12.

31. Balakrishnan B, Tzafriri AR, Seifert P, Groothuis A, Rogers C, Edelman ER. Strut position, blood flow, and drug deposition: implications for single and overlapping drug-eluting stents. Circulation. 2005;111(22):2958-2965.

32. Balakrishanan B, Dooley JF, Kopia G, Edelman ER. Intravascular drug release kinetics dictate arterial drug deposition, retention, and distribution. J Control Release. 2007;123(2):100-108.

33. Sgueglia GA, Chevalier B. Kissing balloon inflation in percutaneous coronary interventions. JACC Cardiovasc Interv. 2012;5(8):803-811.

34. Schwartz RS, Huber KC, Murphy JG, et al. Restenosis and the proportional neointimal response to coronary artery injury: results in a porcine model. J Am Coll Cardiol. 1992;19(2):267-274.

35. Kornowski R, Hong MK, Tio FO, Bramwell O, Wu H, Leon MB. In-stent restenosis: contributions of inflammatory responses and arterial injury to neointimal hyperplasia. J Am Coll Cardiol. 1998;31(1):224-230.

36. Suzuki T, Kopia G, Hayashi S, et al. Stent-based delivery of sirolimus reduces neointimal formation in a porcine coronary model. Circulation. 2001;104(10):1188-1193.
37. Steffel J, Latini RA, Akhmedov A, et al. Rapamycin, but not FK-506, increases endothelial tissue factor expression: implications for drugeluting stent design. Circulation. 2005;112(13):2002-2011.

38. Camici GG, Steffel J, Amanovic I, et al. Rapamycin promotes arterial thrombosis in vivo: implications for everolimus and zotarolimus eluting stents. Eur Heart J. 2010;31(2):236-242.

39. Otsuka F, Finn AV, Yazdani SK, Nakano M, Kolodgie FD, Virmani R. The importance of the endothelium in atherothrombosis and coronary stenting. Nat Rev Cardiol. 2012;9:439-453.

40. Napoli KL, Wang ME, Stepkowski SM, Kahan BD. Distribution of sirolimus in rat tissue. Clin Biochem. 1997;30(2):135-142.

41. Barbato E, Salinger-Martinovic S, Sagic D, et al. A first-in-man clinical evaluation of Ultimaster, a new drug-eluting coronary stent system: CENTURY study. EuroIntervention. 2015;11(5):541-548.

42. Stojkovic S, Neskovic AN, Mehmedbegovic Z, et al. Reduced sirolimus systemic exposure and improved bioresorbable polymer properties: new allies for the treatment of patients with coronary artery disease. Fundam Clin Pharmacol. 2015;29(1):95-105.

43. Chen YW, Smith ML, Sheets M, et al. Zotarolimus, a novel sirolimus analogue with potent anti-proliferative activity on coronary smooth muscle cells and reduced potential for systemic immunosuppression. J Cardiovasc Pharmacol. 2007;49(4):228-235.

44. Wessely R, Blaich B, Belaiba RS, et al. Comparative characterization of cellular and molecular anti-restenotic profiles of paclitaxel and sirolimus. Implications for local drug delivery. Thromb Haemost. 2007;97(6):1003-1012.

45. Gao RL, Xu B, Lansky AJ, et al; TARGET I Investigators. A randomised comparison of a novel abluminal groove-filled biodegradable polymer sirolimus-eluting stent with a durable polymer everolimus-eluting stent: clinical and angiographic follow-up of the TARGET I trial. EuroIntervention. 2013;9:75-83.
Medical Devices: Evidence and Research

\section{Publish your work in this journal}

Medical Devices: Evidence and Research is an international, peerreviewed, open access journal that focuses on the evidence, technology, research, and expert opinion supporting the use and application of medical devices in the diagnosis, treatment and management of clinical conditions and physiological processes. The identification of novel

\section{Dovepress}

devices and optimal use of existing devices which will lead to improved clinical outcomes and more effective patient management and safety is a key feature. The manuscript management system is completely online and includes a quick and fair peer-review system. Visit http://www. dovepress.com/testimonials.php to read real quotes from authors. 